\title{
UNE ESTIMATION DE LA DIMENSION DE KRULL DES ANNEAUX DE DÉFORMATIONS EN RAMIFICATION INCOMPLĖTE
}

\author{
par
}

Christian Maire

\begin{abstract}
Résumé. - Nous étudions la dimension de Krull des anneaux de déformations de représentations galoisiennes quand la ramification en $p$ peut-être incomplète. Sous certaines conditions, nous donnons une minoration non triviale de cette dimension.
\end{abstract}

Abstract. - We study the Krull dimension of deformation rings associated to Galois representation, with ramification restricted to (not necessarily all) places above $p$. Under some conditions we give a non trivial minoration of this dimension.

\section{Table des matières}

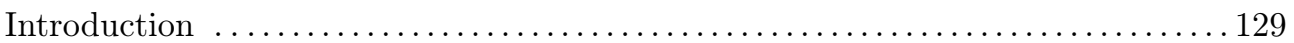

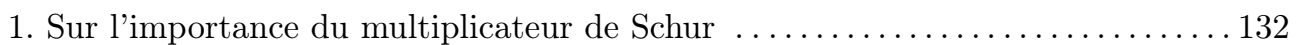

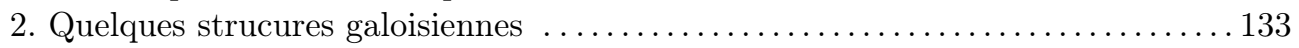

3. Sur la dimension de Krull de l'anneau $\mathrm{R}_{S}^{T} \ldots \ldots \ldots \ldots \ldots \ldots \ldots \ldots \ldots \ldots \ldots$

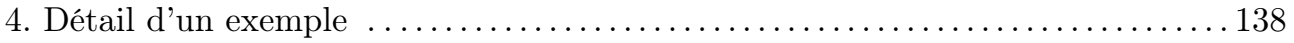

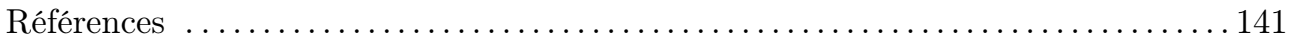

\section{Introduction}

Soient $\mathbf{K}$ un corps de nombres et $S$ un ensemble fini de places finies de $\mathbf{K}$. Pour simplifier, supposons que $S$ ne contient que des places au-dessus de $p$.

Après s'être fixé une clôture algébrique $\overline{\mathbf{K}}$ de $\mathbf{K}$, soit

$$
\bar{\rho}: \operatorname{Gal}\left(\mathbf{K}_{S} / \mathbf{K}\right) \rightarrow \mathrm{Gl}_{2}\left(\mathbf{F}_{\mathrm{p}}\right)
$$

une représentation continue du groupe de Galois $G_{S}:=\operatorname{Gal}\left(\mathbf{K}_{S} / \mathbf{K}\right)$ de la plus grande extension galoisienne $\mathbf{K}_{S}$ de $\mathbf{K}$ pour laquelle les places finies en dehors de $S$ y sont non-ramifiées. Notons par $\mathbf{L}$ l'extension galoisienne maximale de $\mathbf{K}$ fixée par $\operatorname{ker}(\bar{\rho}) ; \operatorname{Gal}(\mathbf{L} / \mathbf{K}) \simeq \Delta=\operatorname{Im}(\bar{\rho})$.

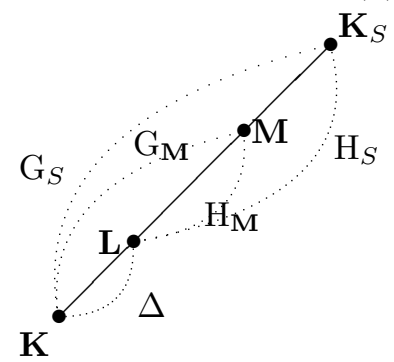

Classification mathématique par sujets (2000). - Primary 11R37; Secondary 11R34.

Mots clefs. - ramification restreinte, anneaux de déformations galoisiennes.

Ce travail a été rédigé lors de mon séjour à l'École Polytechnique de Lausanne, durant l'été 2004. Je remercie Éva Bayer pour son invitation. Je remercie également Christophe Delaunay et Thong Nguyen Quang Do pour leurs remarques. 
Pour une extension galoisienne $\mathbf{M}$ de $\mathbf{K}$ contenue dans $\mathbf{K}_{S}$ et contenant $\mathbf{L}$, posons $\mathrm{H}_{\mathbf{M}}=\operatorname{Gal}(\mathbf{M} / \mathbf{L})$ et $\mathrm{G}_{\mathbf{M}}=\operatorname{Gal}(\mathbf{M} / \mathbf{K})$. La surjection canonique $\mathrm{G}_{S} \rightarrow \mathrm{G}_{\mathbf{M}}$ permet alors à la représentation $\bar{\rho}$ de se factoriser à travers $G_{\mathbf{M}}$.

Soit $\mathcal{C}$ la catégorie des anneaux locaux $\left(\mathrm{R}, \mathfrak{m}_{\mathrm{R}}\right)$ noetherien complet de corps résiduel $\mathbb{F}_{p}$. Si $\mathrm{R}$ est un élément de $\mathcal{C}$ et $\rho: \mathrm{G}_{\mathbf{M}} \rightarrow \mathrm{Gl}_{2}(\mathrm{R})$ une représentation continue de $\mathrm{G}_{\mathbf{M}}$, on dit que celle-ci est un relèvement de la représentation $\bar{\rho}$ si le diagramme suivant est commutatif

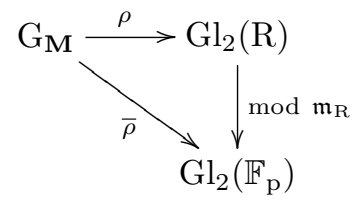

Si $\rho^{\prime}: \mathrm{G}_{\mathbf{M}} \rightarrow \mathrm{Gl}_{2}(\mathrm{R})$ est une autre représentation de $\mathrm{G}_{\mathbf{M}}$, les représentations $\rho$ et $\rho^{\prime}$ sont dites strictement équivalentes si elles sont conjuguées par un élément de $\operatorname{ker}\left(\mathrm{Gl}_{2}(\mathrm{R}) \stackrel{\bmod \mathfrak{m}_{\mathrm{R}}}{\longrightarrow} \mathrm{Gl}_{2}\left(\mathbb{F}_{\mathrm{p}}\right)\right)$. Une classe de conjuguaison d'une telle représentation $\rho$ est appelée une déformation de $\bar{\rho}$ à $R$.

Comme $S$ est fini, des travaux de Mazur [15] montrent l'existence d'un élément $\mathrm{R}_{\mathbf{M}}$ de $\mathcal{C}$ et d'une représentation verselle $\rho_{\mathbf{M}}: \mathrm{G}_{\mathbf{M}} \rightarrow \mathrm{Gl}_{2}\left(\mathrm{R}_{\mathbf{M}}\right)$ relevant la représentation $\bar{\rho}$. Tout relèvement $\rho^{\prime}: \mathrm{G}_{\mathbf{M}} \rightarrow \mathrm{Gl}_{2}(\mathrm{R})$ de $\bar{\rho}$, avec $\mathrm{R} \in \mathcal{C}$, se factorise à travers $\rho_{\mathbf{M}}$, c'est à dire qu'il existe un morphisme d'anneau local $\varphi: \mathrm{R}_{\mathbf{M}} \rightarrow \mathrm{R}$ où la composée $\varphi \circ \rho_{\mathbf{M}}$ produit un relèvement de $\bar{\rho}$ strictement équivalent à $\rho^{\prime}$. L'anneau $\mathrm{R}_{\mathbf{M}}$ est unique, et est appelé anneau de déformation de la représentation $\bar{\rho}$ (associée à l'extension $\mathbf{M} / \mathbf{K}$ ). Lorsque $\bar{\rho}$ est absolument irréductible (non triviale) la représentation $\rho_{\mathbf{M}}$ est de plus universelle, c'est à dire que la donnée de $\rho_{\mathbf{M}}$ détermine $\varphi$.

Si l'on note par Ad la représentation adjointe associée à $\bar{\rho}$, Mazur a montré que la dimension de Krull de l'anneau $\mathrm{R}_{\mathbf{M}} / p \mathrm{R}_{\mathbf{M}}$ est minorée par la quantité $d_{p} H^{1}\left(\mathrm{G}_{\mathbf{M}}, \mathrm{Ad}\right)-d_{p} H^{2}\left(\mathrm{G}_{\mathbf{M}}, \mathrm{Ad}\right)$. En fait, l'anneau de déformation $\mathrm{R}_{\mathbf{M}}$ est de la forme $\mathbb{Z}_{p}\left[\left[T_{1}, \cdots, T_{r}\right]\right] / I$, où $r=\operatorname{dim}_{\mathbf{F}_{p}} H^{1}\left(\mathrm{G}_{\mathbf{M}}, \mathrm{Ad}\right)$. L'idéal $I$ est trivial dès lors que le groupe de cohomologie $H^{2}\left(\mathrm{G}_{\mathbf{M}}, \mathrm{Ad}\right)$ l'est. Si tel est le cas (i.e. $\left.I=(0)\right)$, on dit que la déformation $\rho_{\mathbf{M}}$ est sans obstruction.

Remarquons que comme le noyau de la restriction $\mathrm{Gl}_{2}(\mathrm{R}) \rightarrow \mathrm{Gl}_{2}\left(\mathbb{F}_{\mathrm{p}}\right)$ est un pro-p-groupe (voir par exemple [1] $)$, tout relèvement de $\bar{\rho}: \mathrm{G}_{\mathbf{M}} \rightarrow \mathrm{Gl}_{2}\left(\mathbb{F}_{\mathrm{p}}\right)$ se factorise à travers la plus grande pro- $p$-extension $\mathbf{M}(p)$ de $\mathbf{L}$ contenue dans $\mathbf{M}$.

Fixons $T$ un second ensemble fini de places finies de $\mathbf{K}$. Assurons-nous que $T$ et $S$ sont disjoints. Nous nous intéressons au cas où le corps $\mathbf{M}$ correspond à $\mathbf{L}_{S}^{T}(p)$, où $\mathbf{L}_{S}^{T}(p)$ est la plus grande extension de $\mathbf{L}$ contenue dans $\mathbf{K}_{S}$ pour laquelle l'ensemble des places au-dessus des places de $T$ y sont totalement décomposées. Nous noterons par $\mathrm{R}_{S}^{T}$ son anneau de déformation et par $\rho_{S}^{T}$ : $\operatorname{Gal}\left(\mathbf{L}_{S}^{T}(p) / \mathbf{K}\right) \rightarrow \mathrm{Gl}_{2}\left(\mathrm{R}_{\mathrm{S}}^{\mathrm{T}}\right)$ une déformation verselle de $\bar{\rho}$ associée à cette extension.

Posons $\mathrm{P}_{S}^{T}=\mathrm{H}_{\mathbf{L}_{S}^{T}(p)}=\operatorname{Gal}\left(\mathbf{L}_{S}^{T}(p) / \mathbf{L}\right)$ le groupe de Galois de l'extension $\mathbf{L}_{S}^{T}(p) / \mathbf{L}$.

Lorsque $S$ contient l'ensemble des places au-dessus de $p$, grâce à la dualité de Poitou-Tate (cf. par exemple [9], [17]), Mazur a montré comment obtenir une minoration de la dimension de Krull de l'anneau $\mathrm{R}_{S}$. Ce point de départ a ensuite été utilisé par de nombreux auteurs (Mazur [15], Boston [3], Boston et Mazur [4], Ramakrishna [18], Böckle [1] [2], Böckle et Mézard [16], Gouvêa [6], ‥). Dans ce cas (ici $T=\emptyset$ ), la différence $d_{p} H^{1}\left(\mathrm{G}_{S}, \mathrm{Ad}\right)-d_{p} H^{2}\left(\mathrm{G}_{S}\right.$, Ad) est parfaitement connue. Avant d'en donner une évaluation, donnons une définition.

Définition 0.1. - Une place infinie $v$ de $\mathbf{K}$ est dite impaire (relativement à $\bar{\rho}$ ) si le déterminant de $\bar{\rho}\left(s_{v}\right)$ est -1 , où $s_{v}$ est un générateur du sous-groupe de décomposition $\Delta_{v}$ de $v$ dans $\Delta$. 
La place $v$ est dite paire sinon.

Notons par $\kappa_{1}$ le nombre de places infinies $v$ de $\mathbf{K}$ en lesquelles la représentation $\bar{\rho}_{\mid \Delta_{v}}$ est impaire et par $\kappa_{0}$ le nombre de places infinies de $\mathbf{K}$ en lesquelles la représentation $\bar{\rho}_{\mid \Delta_{v}}$ est paire. Il vient :

$$
\begin{aligned}
d_{p} H^{1}\left(\mathrm{G}_{S}, \mathrm{Ad}\right)-d_{p} H^{2}\left(\mathrm{G}_{S}, \mathrm{Ad}\right)= & d_{p} H 0\left(\mathrm{G}_{S}, \mathrm{Ad}\right)+4\left(r_{1}+2 r_{2}\right) \\
& -4 \kappa_{0}-2 \kappa_{1} \\
\geq & 1+4\left(r_{1}+2 r_{2}\right)-4 \kappa_{0}-2 \kappa_{1} \\
= & 1+2 \kappa_{1}+4 r_{2},
\end{aligned}
$$

où $\left(r_{1}, r_{2}\right)$ est la signature du corps $\mathbf{K}$ (à noter que le $\mathrm{G}_{S}$-module Ad contient au moins un sousespace non réduit à $\{0\}$ avec action triviale, les matrices diagonales).

Malheureusement, une telle démarche n'est plus possible dès que $S$ ne contient plus à priori toutes les places au-dessus de $p$ (c'est ce que l'on appelle la situation $p$-ramification incomplète). Néanmoins, lorsque $(\# \Delta, p)=1$, nous donnons ici une minoration non triviale de la dimension de Krull de l'anneau $\mathrm{R}_{S}^{T} / p \mathrm{R}_{S}^{T}$ dès lors que le poids $\delta_{S}$ de $S$ est suffisamment grand.

Définition 0.2. - Nous définissons le poids $\delta_{S}$ de $S$ comme étant la quantité

$$
\delta_{S}=\sum_{v \in S}\left[\mathbf{K}_{v}: \mathbb{Q}_{p}\right]
$$

Définition 0.3. - Supposons $\Delta$ d'ordre premier à $p$. Soit $v$ une place de $\mathbf{K}$. Notons par $\Delta_{v}$ le groupe de décomposition de $v$ dans $\mathbf{L} / \mathbf{K}$. Soit $\overline{\rho_{v}}:=\bar{\rho}_{\Delta_{v}}$ la restriction de $\bar{\rho}$ à $\Delta_{v}$. Notons alors par $\tau_{v}$ le nombre de fois qu'apparaît la représentation triviale dans la décomposition du $\Delta_{v}$-module Ad. Ou encore : $\tau_{v}=\langle\operatorname{Ad}, \mathbf{1}\rangle_{\mid \Delta_{v}}$. Posons $\kappa_{v}=4-\tau_{v}$, puis pour $\Sigma$ un ensemble de places de $\mathbf{K}$,

$$
\kappa_{\Sigma}=\sum_{v \in \Sigma} \kappa_{v}
$$

Remarque 0.4. - Notons par $P l_{\infty}$ l'ensemble des places archimédiennes de K. Alors $\kappa_{P l_{\infty}}=$ $2 \kappa_{1}$.

Démonstration. - En effet, une place $v$ est impaire si et seulement si $\tau_{v}=2$. Sinon $\tau_{v}=4$. $\diamond$

On en arrive à l'énoncé du résultat principal.

Théorème 0.5. - Supposons \# $\Delta$ premier à $p$ et $\delta_{S} \geq r_{1}+r_{2}+|T|$. Alors

$$
\operatorname{dimkrull}\left(\mathrm{R}_{S}^{T} / p \mathbf{R}_{S}^{T}\right) \geq 1+2 \kappa_{1}+\kappa_{T}+4\left(\delta_{S}-\left(r_{1}+r_{2}+|T|\right)\right) .
$$

(Si $\operatorname{Im}(\bar{\rho})$ est un sous-groupe de Cartan l'inégalité est stricte.)

Corollaire 0.6. - Plaçons nous sous les conditions du théorème précédent. Supposons de plus que pour toute place $v$ de $T, \Delta_{v}=\{1\}$. Alors

$$
\operatorname{dimkrull}\left(\mathbf{R}_{S}^{T} / p \mathbf{R}_{S}^{T}\right) \geq 1+2 \kappa_{1}+4\left(\delta_{S}-\left(r_{1}+r_{2}+|T|\right)\right) .
$$

(Si $\operatorname{Im}(\bar{\rho})$ est un sous-groupe de Cartan l'inégalité est stricte.) 
Notons que la condition $\delta_{S} \geq r_{1}+r_{2}+|T|$ implique l'existence d'une $\mathbb{Z}_{p}$-extension de $\mathbf{L}$ contenue dans $\mathbf{L}_{S}^{T}(p) / \mathbf{L}$.

Le théorème 0.5 se montre en utilisant l'action semi-simple de $\Delta$ sur les groupes de cohomologie $H^{i}\left(\mathrm{P}_{S}^{T}, \mathrm{Ad}\right), i=1,2$. Nous explicitons cette action galoisienne dans le paragraphe 3 . Dans la paragraphe 4, nous montrons le théorème principal. Enfin, le dernier paragraphe est consacré à l'étude d'un exemple se situant dans la lignée de l'exemple étudié par Mazur dans [15].

À noter qu'ici nous ne regardons l'anneau $\mathrm{R}_{S}^{T}$ que sous un angle purement arithmétique.

Notations. Pour un $\mathbb{Z}_{p}$-module de type fini $M$, nous désignerons par

$$
\operatorname{Tor}(M)=\left\{m \in M, \exists k \in \mathbb{Z}_{p}, m^{k}=1\right\}
$$

le sous-module de torsion de $M$, et par

$$
\operatorname{Tor}_{p}(M)=\left\{m \in M, m^{p}=1\right\},
$$

le sous-module de $p$-torsion de $M$.

Pour un corps $\mathbf{F}, \mu_{p}(\mathbf{F})$ désignera le groupe des racines $p$-èmes de l'unité contenues dans $\mathbf{F}$ et pour un groupe abélien localement compact $G$, son dual de Pontryagin sera noté $G^{*}$.

\section{Sur l'importance du multiplicateur de Schur}

Nous allons montrer le rôle crucial que joue le multiplicateur de Schur dans le calcul de la dimension de Krull de l'anneau $\mathrm{R}_{\mathbf{M}}$. Commençons par un premier lemme :

Lemme 1.1. - La suite exacte $1 \longrightarrow \mathrm{H}_{\mathbf{M}} \longrightarrow \mathrm{G}_{\mathbf{M}} \longrightarrow \Delta \longrightarrow 1$ fournit l'isomorphisme de $\Delta$-modules suivant:

$$
H^{i}\left(\mathrm{H}_{\mathbf{M}}, \mathrm{Ad}\right) \simeq H^{i}\left(\mathrm{H}_{\mathbf{M}}, \mathbb{F}_{p}\right) \otimes \mathrm{Ad}
$$

Démonstration. - Il suffit de noter que $\mathrm{H}_{\mathbf{M}}$ agit trivialement sur $\mathrm{Ad}, \mathbb{F}_{p}$-espace vectoriel de dimension 4, puis que l'isomorphisme souhaité est obtenu via la composition des isomorphismes suivants :

$$
H^{i}\left(\mathrm{H}_{\mathbf{M}}, \mathbb{F}_{p}\right) \otimes \mathrm{Ad} \stackrel{\sim}{\longrightarrow} \bigoplus_{j=1}^{4} H^{n}\left(\mathrm{H}_{\mathbf{M}}, \mathbb{F}_{p}\right) e_{j} \stackrel{\sim}{\longrightarrow} H^{n}\left(\mathrm{H}_{\mathbf{M}}, \mathrm{Ad}\right)
$$

et que ceux-ci sont bien des isomorphismes de $\Delta$-modules (où $\left(e_{1}, \cdots, e_{4}\right)$ est une $\mathbb{F}_{p}$-base de $\mathrm{Ad}$ ). $\diamond$

Lemme 1.2. - Soit G un pro-p-groupe de type fini. Alors

$$
d_{p} H^{1}\left(\mathrm{G}, \mathbb{F}_{p}\right)-d_{p} H^{2}\left(\mathrm{G}, \mathbb{F}_{p}\right)=d_{p} \mathrm{Fr}_{\mathbb{Z}_{p}} \mathrm{G}-d_{p} H_{2}\left(\mathrm{G}, \mathbb{Z}_{p}\right),
$$

où $\mathrm{Fr}_{\mathbb{Z}_{p}} \mathrm{G}$ désigne la partie $\mathbb{Z}_{p}$-libre de $\mathrm{G}^{a b}$. Si de plus $H_{2}\left(G, \mathbb{Z}_{p}\right)$ est trivial alors, $H^{2}\left(G, \mathbb{F}_{p}\right) \simeq$ $\operatorname{Tor}_{p}\left(G^{a b}\right)$.

Démonstration. - C'est bien connu, cela résulte de la suite exacte courte :

$$
1 \longrightarrow \mathbb{F}_{p} \longrightarrow \mathbb{Q}_{p} / \mathbb{Z}_{p} \stackrel{p}{\longrightarrow} \mathbb{Q}_{p} / \mathbb{Z}_{p} \longrightarrow 1 \text {. }
$$


Lemme 1.3. - Supposons \# $\Delta$ premier à $p$.

(i) On a, pour tout $i \geq 1, H^{i}\left(\mathrm{G}_{\mathbf{M}}, \mathrm{Ad}\right) \simeq\left[H^{i}\left(\mathrm{H}_{\mathbf{M}}, \mathbb{F}_{p}\right) \otimes \mathrm{Ad}\right]^{\Delta}$.

(ii) $H^{1}\left(\mathrm{G}_{\mathbf{M}}, \mathrm{Ad}\right) \simeq\left[\left(\mathrm{H}_{\mathbf{M}}^{a b}\right)^{*} \otimes \mathrm{Ad}\right]^{\Delta}$, où $\mathrm{H}_{\mathbf{M}}^{a b}:=\mathrm{H}_{\mathbf{M}} /\left[\mathrm{H}_{\mathbf{M}}, \mathrm{H}_{\mathbf{M}}\right]$.

(iii) Si le groupe $H^{2}\left(\mathrm{H}_{\mathbf{M}}, \mathbb{Q}_{p} / \mathbb{Z}_{p}\right)$ est trivial, il vient alors :

$$
H^{2}\left(\mathrm{G}_{\mathbf{M}}, \mathrm{Ad}\right) \simeq\left[\left(\operatorname{Tor}_{p}\left(\mathrm{H}_{\mathbf{M}}^{a b}\right)\right)^{*} \otimes \mathrm{Ad}\right]^{\Delta} .
$$

Démonstration. - (i) Comme \# $\Delta$ est premier à $p$, la suite spectrale au niveau supérieur donne l'isomorphisme entre $H^{i}\left(\mathrm{G}_{\mathbf{M}}, A\right)$ et $H^{i}\left(\mathrm{H}_{\mathbf{M}}, A\right)^{\Delta}$ et ce pour tout $\mathrm{G}_{\mathbf{M}}$-module discret $p$-primaire de torsion $A$. Il suffit ensuite d'utiliser le lemme 1.1 .

(ii) C'est un cas particulier du point précédent.

(iii) Sous l'hypothèse $H^{2}\left(\mathrm{H}_{\mathbf{M}}, \mathbb{Q}_{p} / \mathbb{Z}_{p}\right)=0$, on a $\operatorname{Tor}_{p}\left(\mathrm{H}_{\mathbf{M}}^{a b}\right) \simeq H_{2}\left(\mathrm{H}_{\mathbf{M}}, \mathbb{F}_{p}\right)$. Il suffit ensuite d'utiliser le point (i). $\diamond$

Ainsi, ces trois lemmes montrent que pour avoir une minoration non triviale de la dimension de Krull de $R_{\mathbf{M}}$, il est souhaitable que d'une part le corps $\mathbf{L}$ ait au moins une $\mathbb{Z}_{p}$-extension contenue dans $\mathbf{M} / \mathbf{L}$ (ou encore que $\mathrm{Fr}_{\mathbb{Z}_{p}} \mathrm{H}_{\mathbf{M}}$ soit non trivial) et que d'autre part l'on puisse contrôler le multiplicateur de Schur $H_{2}\left(\mathrm{H}_{\mathbf{M}}, \mathbb{Z}_{p}\right)$ du groupe $\mathrm{H}_{\mathbf{M}}$.

Quand $S$ contient l'ensemble des places au-dessus de $p$ et pour $\mathbf{M}=\mathbf{L}_{S}(p)$, ces deux quantités sont intimement liées. En fait, la conjecture de Leopoldt pour le couple $(\mathbf{L}, p)$, donne exactement le rang de $\mathrm{Fr}_{\mathbb{Z}_{p}} \mathrm{H}_{S}$, et cette conjecture équivaut à la trivialité du multiplicateur de Schur du groupe $\operatorname{Gal}\left(\mathbf{L}_{S}(p) / \mathbf{L}\right)$.

Maintenant, lorsque $S$ ne contient pas toutes les places au-dessus de $p$, il est quand même possible d'obtenir des informations sur ce multiplicateur de Schur. Comme pour le cas où $S$ contient l'ensemble des places au-dessus de $p$, nous verrons (cf. corollaire 2.4) que le multiplicateur de Schur de ce groupe $\operatorname{Gal}\left(\mathbf{L}_{S}(p) / \mathbf{L}\right)$ est aussi dans ce cas lié à un problème d'indépendance $p$-adique.

\section{Quelques strucures galoisiennes}

Pour toute la suite, nous supposons que l'ensemble $S$ est non vide et qu'il ne contient que des places finies $v$ au-dessus de $p$.

2.1. Les notations. - Pour une place $v$ de $\mathbf{K}$, notons par $\Delta_{v}=\Delta_{w}$ le groupe de décomposition dans $\mathbf{L} / \mathbf{K}$ d'une place quelconque $w$ de $\mathbf{L}$ au-dessus de $v$. Pour un ensemble $E$ de places de $\mathbf{K}$, nous notons par $E(\mathbf{L})$ l'ensemble des places $\mathbf{L}$ au-dessus des places de $E$.

Soit

$$
\amalg_{S}^{T}(\mathbf{L})=\left\{x \in \mathbf{L}^{\times}, \mathrm{v}_{w}(x) \equiv 0 \bmod p, \forall w \notin T(\mathbf{L}) ; x \in \mathbf{L}_{w}^{p}, \forall w \in S(\mathbf{L})\right\} / \mathbf{L}^{\times p}
$$

le groupe de Shafarevich du corps $\mathbf{L}$ associé aux ensembles $S$ et $T$.

Pour $w$ une place finie de $\mathbf{L}$, notons $\mathfrak{U}_{w}$ le sous-groupe des unités principales du complété $\mathbf{L}_{w}$ de $\mathbf{L}$ en $w$. Soit $\mathfrak{U}_{S}=\prod_{w \in S(\mathbf{L})} \mathfrak{U}_{w}$ et soit

$$
\Psi_{S}^{T}=\mathbb{Z}_{p} \otimes \mathrm{E}_{\mathbf{L}}^{T} \rightarrow \mathfrak{U}_{S}
$$

le plongement des $T(\mathbf{L})$-unités $\mathrm{E}_{\mathbf{L}}^{T}$ de $\mathbf{L}$ dans le produit des complétés des places de $S(\mathbf{L})$ (qui rappelons-le sont toutes au-dessus de $p$ ).

Fixons $\mathbb{C}_{p}$ le complété d'une clôture algébrique de $\mathbb{Q}_{p}$. Lorsque $w$ est une place de $S(\mathbf{L})$, notons par $\log _{w}$ le logarithme $p$-adique associé à la place $w$. Pour $x=\left(x_{1}, \cdots, x_{s}\right) \in \mathfrak{U}_{S}$, nous posons

$$
\log (x)=\left(\log _{w_{1}}\left(x_{1}\right), \cdots, \log _{w_{s}}\left(x_{s}\right)\right) \in \mathbb{C}_{p}^{s} .
$$




\subsection{Les principales estimations. -}

Supposons \# $\#$ premier à $p$.

Pour un $\mathbb{Z}_{p}[\Delta]$-module $M$ et un caractère $\mathbb{C}_{p}[\Delta]$-irréductible $\varphi, M_{\varphi}$ désignera la $\varphi$-partie de ce module et $r_{\varphi}(M)$ le $\varphi$-rang de $M$. Rappelons que $M_{\varphi}$ est vu comme module sur l'anneau local $\mathbb{Z}_{p}[\varphi]$. Si $\psi$ et $\psi^{\prime}$ sont deux caractères de $\mathbb{Z}_{p}[\Delta]$, nous noterons par $\left\langle\psi, \psi^{\prime}\right\rangle$ le produit scalaire entre ceux-ci. Si $M$ est un $\mathbb{Z}_{p}[\Delta]$-module, on identifiera $M$ avec son caractère. Ainsi, par exemple, si $\varphi$ est $\mathbb{C}_{p}$-irréductible, $r_{\varphi}(M)=\langle M, \varphi\rangle$.

Pour un sous-groupe $\mathrm{H}$ de $\Delta$ et un $\mathrm{H}$-module $M, \operatorname{Ind}_{\mathrm{H}}^{\Delta}(M)$ désignera le $\Delta$-module induit de $\mathrm{H}$ à $\Delta$ obtenu à partir de $M$. On rappelle la formule de réciprocité :

$$
<M, \operatorname{Ind}_{\mathrm{H}}^{\Delta} N>=<M, N>_{\mid \mathrm{H}} .
$$

Si $\chi$ est le caractère du $\Delta$-module $M$, le caractère du dual $M^{*}$ de $M$ sera noté $\chi^{*}$. On rappelle que pour tout $\sigma$ de $\Delta$, on a $\chi^{*}(\sigma)=\chi\left(\sigma^{-1}\right)$.

Nous noterons par $\mathbf{1}$ le caractère de la représentation triviale (ou unité) : $\mathbf{1}(\sigma)=1$, pour tout $\sigma \in \Delta$.

Soit donc $\mathrm{P}_{S}^{T}=\operatorname{Gal}\left(\mathbf{L}_{S}^{T}(p) / \mathbf{L}\right)$. La structure du $\Delta$-module $\mathrm{P}_{S}^{T, a b}:=\mathrm{P}_{S}^{T} /\left[\mathrm{P}_{S}^{T}, \mathrm{P}_{S}^{T}\right]$ est parfaitement connue :

Proposition 2.1 (Gras [7]). — Soit $\varphi$ un caractère $\mathbb{C}$-irréductible de $\Delta$. Alors

$$
r_{\varphi}\left(\mathrm{P}_{S}^{T, a b}\right)=r_{\varphi}\left(\amalg_{S}^{T}(\mathbf{L})\right)-r_{\varphi}\left(\mathrm{E}_{\mathbf{L}}^{T}\right)+\sum_{v \in S} \delta_{v} r_{\varphi} \operatorname{Ind}_{\Delta_{v}}^{\Delta} \mu_{p}\left(\mathbf{L}_{v}\right)+\varphi(1)\left(\delta_{S}+\delta_{2, p} r_{1}(\mathbf{L})\right),
$$

où $\delta_{S}=\sum_{v \in S}\left[\mathbf{K}_{v}: \mathbb{Q}_{p}\right]$, où $\delta_{v}=\delta_{w}=1$ si $\mathbf{L}_{w}$ contient les racines $p$-èmes de l'unité, 0 sinon, où $r_{1}(\mathbf{L})$ est le nombre de places réelles de $\mathbf{L}$ et où $\delta_{2, p}$ est le symbole de Kronecker.

En ce qui concerne le $\varphi$-rang du $\Delta$-module $H_{2}\left(\mathrm{P}_{S}^{T}, \mathbb{F}_{p}\right)$, il est possible d'en donner une majoration :

Proposition 2.2. - Soit $\varphi$ un caractère $\mathbb{C}$-irréductible de $\Delta$. Alors

$$
r_{\varphi}\left(H_{2}\left(\mathrm{P}_{S}^{T}, \mathbb{F}_{p}\right)\right) \leq r_{\varphi}\left(\amalg_{S}^{T}(\mathbf{L})\right)-r_{\varphi} \mu_{p}(\mathbf{L})+\sum_{v \in S} \delta_{v} r_{\varphi} \operatorname{Ind}_{\Delta_{v}}^{\Delta} \mu_{p}\left(\mathbf{L}_{v}\right)+\delta_{2, p} \varphi(1) r_{1}(\mathbf{L})
$$

Démonstration. - On reprend la preuve classique permettant de donner une majoration du $p$-rang de $H^{2}\left(\mathrm{P}_{S}^{T}, \mathbb{F}_{p}\right)$ tout en faisant ressortir l'action de $\Delta$ (voir [12], [11], [7], [13] ...)

Pour commencer un lemme (cf. [12], [17]) :

Lemme 2.3. - Soit $\bar{G}_{\mathbf{L}}(p)$ (respectivement $\bar{G}_{w}(p)$ ) le plus grand pro-p-quotient du groupe de Galois absolu $\bar{G}_{\mathbf{L}}$ (resp. $\left.\bar{G}_{w}\right)$ de $\mathbf{L}\left(\right.$ resp. $\left.\mathbf{L}_{w}\right)$. On a la suite de $\Delta$-modules :

$$
1 \longrightarrow H^{2}\left(\bar{G}_{\mathbf{L}}(p), \mathbb{F}_{p}\right) \stackrel{\operatorname{Loc}}{\longrightarrow} \oplus_{w} H^{2}\left(\bar{G}_{w}(p), \mathbb{F}_{p}\right) \longrightarrow\left(\left(\mu_{p}\right)^{\bar{G}_{\mathbf{L}}(p)}\right)^{*} \longrightarrow 1 .
$$

Ensuite, on sait qu'il existe un $\Delta$-morphisme

$$
\operatorname{ker}\left(H^{2}\left(\mathrm{P}_{S}^{T}, \mathbb{F}_{p}\right) \stackrel{\operatorname{Inf}}{\rightarrow} H^{2}\left(\bar{G}_{\mathbf{L}}(p), \mathbb{F}_{p}\right)\right) \hookrightarrow\left(\amalg_{S}^{T}(\mathbf{L})\right)^{*}
$$

obtenu à partir du symbole de réciprocité local (cf. [12]). Ainsi,

$$
\operatorname{ker}(\operatorname{Loc} \circ \operatorname{Inf}) \hookrightarrow\left(\amalg_{S}^{T}(\mathbf{L})\right)^{*},
$$


où Inf : $H^{2}\left(\mathrm{P}_{S}^{T}, \mathbb{F}_{p}\right) \rightarrow H^{2}\left(\bar{G}_{\mathbf{L}}(p), \mathbb{F}_{p}\right)$. Comme les places en dehors de $S$ et de l'infini sont non ramifiées dans $\mathbf{L}_{S}^{T}(p) / \mathbf{L}$,

$$
\operatorname{Loc} \circ \operatorname{Inf}\left(H^{2}\left(\mathrm{P}_{S}^{T}, \mathbb{F}_{p}\right)\right)=\operatorname{Loc}_{S^{\prime}} \circ \operatorname{Inf}\left(H^{2}\left(\mathrm{P}_{S}^{T}, \mathbb{F}_{p}\right)\right),
$$

où $\operatorname{Loc}_{S^{\prime}}$ est la projection de Loc sur les composantes en $S(\mathbf{L})$ et en les places réelles de $\mathbf{L}$. D'autre part le morphisme

$$
\bigoplus_{w \in S(\mathbf{L}) \cup P l_{\infty}} H^{2}\left(\bar{G}_{w}(p), \mathbb{F}_{p}\right) \stackrel{\Sigma_{S^{\prime}}}{\rightarrow} \mu_{p}(\mathbf{L})^{*}
$$

est surjectif (se rappeler que par hypothèse, $S$ est non vide) et $\sum_{S^{\prime}} \circ$ Loc $\circ \operatorname{Inf}=0$. En résumé :

$$
\begin{aligned}
r_{\varphi} H^{2}\left(\mathrm{P}_{S}^{T}, \mathbb{F}_{p}\right) & =r_{\varphi} \operatorname{ker}(\operatorname{Loc} \circ \operatorname{Inf})+r_{\varphi} \operatorname{Im}(\operatorname{Loc} \circ \operatorname{Inf}) \\
& \leq r_{\varphi}\left(\amalg_{S}^{T}(\mathbf{L})\right)^{*}+r_{\varphi} \operatorname{Loc}\left(\operatorname{Inf}\left(H^{2}\left(\mathrm{P}_{S}^{T}, \mathbb{F}_{p}\right)\right)\right) \\
& =r_{\varphi}\left(\amalg_{S}^{T}(\mathbf{L})\right)^{*}+r_{\varphi} \operatorname{Loc} S_{S^{\prime}}\left(\operatorname{Inf}\left(H^{2}\left(\mathrm{P}_{S}^{T}, \mathbb{F}_{p}\right)\right)\right) \\
& \leq r_{\varphi}\left(\amalg_{S}^{T}(\mathbf{L})\right)^{*}+r_{\varphi} \operatorname{ker} \sum_{S^{\prime}} \\
& =r_{\varphi}\left(\amalg_{S}^{T}(\mathbf{L})\right)^{*}+r_{\varphi}\left(\bigoplus_{w \in S(\mathbf{L}) \cup P l_{\infty}} H^{2}\left(\bar{G}_{w}(p), \mathbb{F}_{p}\right)\right)-r_{\varphi} \mu_{p}(\mathbf{L})^{*} .
\end{aligned}
$$

Notons ensuite

$$
\bigoplus_{w \in S(\mathbf{L}) \cup P l_{\infty}} H^{2}\left(\bar{G}_{w}(p), \mathbb{F}_{p}\right)=\bigoplus_{v \in S \cup P l_{\infty}} \operatorname{Ind}_{\Delta_{v}}^{\Delta} H^{2}\left(\bar{G}_{v}(p), \mathbb{F}_{p}\right)
$$

où, dans la seconde somme, on a privilégié le choix d'une place $w$ de $\mathbf{L}$ au-dessus de $v$ et posé $\overline{\mathrm{G}}_{v}(p)=\bar{G}_{w}(p)$.

Pour les places $w$ de $\mathbf{L}$ en lesquelles le groupe $\mu_{p}\left(\mathbf{L}_{w}\right)$ est trivial, on sait que le pro- $p$-groupe $\bar{G}_{w}(p)$ est libre c'est à dire que $H^{2}\left(\bar{G}_{w}(p), \mathbb{F}_{p}\right)=0$. Pour les autres places $w, \bar{G}_{w}(p)$ agit trivialement sur $\mu_{p}\left(\mathbf{L}_{w}\right)$, et ainsi (à l'aide de la suite exacte de Kummer)

$$
\begin{aligned}
H^{2}\left(\bar{G}_{w}(p), \mathbb{F}_{p}\right) \otimes \mu_{p}\left(\mathbf{L}_{w}\right) & \simeq H^{2}\left(\bar{G}_{w}(p), \mu_{p}\left(\mathbf{L}_{w}\right)\right. \\
& \simeq H^{2}\left(\bar{G}_{w}, \mu_{p}\left(\mathbf{L}_{w}\right)\right) \\
& \simeq \operatorname{Tor}_{p}\left(\operatorname{Br}\left(\mathbf{L}_{w}\right)\right) \simeq \mathbb{Z} / p \mathbb{Z},
\end{aligned}
$$

où $\operatorname{Tor}_{p}\left(\operatorname{Br}\left(\mathbf{L}_{w}\right)\right)$ est la $p$-torsion du groupe de Brauer $\operatorname{Br}\left(\mathbf{L}_{w}\right)$ de $\mathbf{L}_{w}$. Tout ceci étant des isomorphismes de $\Delta_{w}$-modules.

Au total, on obtient :

$$
r_{\varphi} H^{2}\left(\mathrm{P}_{S}^{T}(p), \mathbb{F}_{p}\right) \leq r_{\varphi}\left(\amalg_{S}^{T}(\mathbf{L})\right)^{*}+\sum_{v \in S} \delta_{v} r_{\varphi} \operatorname{Ind}_{\Delta_{v}}^{\Delta} \mu_{p}\left(\mathbf{L}_{v}\right)^{*}+\varphi(1) r_{1}(\mathbf{L}) \delta_{2, p}-r_{\varphi} \mu_{p}(\mathbf{L})^{*}
$$

ou encore

$$
r_{\varphi} H_{2}\left(\mathrm{P}_{S}^{T}, \mathbb{F}_{p}\right) \leq r_{\varphi} \amalg_{S}^{T}(\mathbf{L})+\sum_{v \in S} r_{\varphi} \operatorname{Ind}_{\Delta_{v}}^{\Delta} \mu_{p}\left(\mathbf{L}_{v}\right)+\varphi(1) r_{1}(\mathbf{L}) \delta_{2, p}-r_{\varphi} \mu_{p}(\mathbf{L}) .
$$

$\diamond$

Ces propositions permettent d'en déduire le corollaire suivant :

Corollaire 2.4. - (i) $r_{\varphi}\left(H_{2}\left(\mathrm{P}_{S}^{T}, \mathbb{Z}_{p}\right)\right) \leq r_{\varphi}\left(\operatorname{ker}\left(\Psi_{S}^{T}\right)\right)$.

En particulier, le groupe $H^{2}\left(\mathrm{P}_{S}^{T}, \mathbb{Q}_{p} / \mathbb{Z}_{p}\right)$ est trivial dès lors que $\Psi_{S}^{T}$ est injectif.

(ii) $r_{\varphi}\left(\operatorname{Tor}_{p}\left(\mathrm{P}_{S}^{T, a b}\right)\right) \leq r_{\varphi}\left(H_{2}\left(\mathrm{P}_{S}^{T}, \mathbb{F}_{p}\right)\right) \leq r_{\varphi}\left(\operatorname{Tor}_{p}\left(\mathrm{P}_{S}^{T, a b}\right)\right)+r_{\varphi}\left(\operatorname{ker}\left(\Psi_{S}^{T}\right)\right)$.

Ainsi, lorsque $\Psi_{S}^{T}$ est injectif, $\operatorname{Tor}_{p}\left(\mathrm{P}_{S}^{T, a b}\right) \simeq H_{2}\left(\mathrm{P}_{S}^{T}, \mathbb{F}_{p}\right)$, isomorphisme de $\Delta$-modules. 
Démonstration. - La suite exacte

$$
1 \longrightarrow \mathbb{Z}_{p} \longrightarrow \mathbb{Z}_{p} \longrightarrow \mathbb{Z}_{p} / p \mathbb{Z}_{p} \longrightarrow 1
$$

fournit immédiatement l'égalité

$$
r_{\varphi}\left(H_{2}\left(\mathrm{P}_{S}^{T}, \mathbb{Z}_{p}\right) / p\right)=r_{\varphi}\left(H_{2}\left(\mathrm{P}_{S}^{T}, \mathbb{F}_{p}\right)\right)-r_{\varphi}\left(\operatorname{Tor}_{p}\left(\mathrm{P}_{S}^{T, a b}\right)\right) .
$$

(Noter qu'ici tous les $\mathbb{Z}_{p}$-modules sont de type fini.)

La torsion de $\mathrm{P}_{S}^{T, a b}$ étant finie, il vient

$$
\begin{aligned}
r_{\varphi}\left(\operatorname{Tor}_{p}\left(\mathrm{P}_{S}^{T, a b}\right)\right)= & r_{\varphi}\left(\operatorname{Tor}\left(\mathrm{P}_{S}^{T, a b}\right) /\left(\operatorname{Tor}\left(\mathrm{P}_{S}^{T, a b}\right)\right)^{p}\right) \\
= & r_{\varphi}\left(\operatorname{Tor}\left(\mathrm{P}_{S}^{T, a b}\right)\right) \\
= & r_{\varphi}\left(\mathrm{P}_{S}^{T, a b}\right)-\delta_{S} \varphi(1)+r_{\varphi}\left(\operatorname{Im}\left(\Psi_{S}^{T}\right)\right) \\
= & r_{\varphi}\left(\amalg_{S}^{T}(\mathbf{L})\right)-r_{\varphi}\left(\mathrm{E}_{\mathbf{L}}^{T}\right)+\sum_{v \in S} \delta_{v} r_{\varphi} \operatorname{Ind}_{\Delta_{v}}^{\Delta} \mu_{p}\left(\mathbf{L}_{v}\right) \\
& +\varphi(1) \delta_{2, p} r_{1}(\mathbf{L})+r_{\varphi}\left(\operatorname{Im}\left(\Psi_{S}^{T}\right)\right)
\end{aligned}
$$

Par conséquent

$$
r_{\varphi}\left(H_{2}\left(\mathrm{P}_{S}^{T}, \mathbb{Z}_{p}\right)\right) \leq r_{\varphi}\left(\operatorname{Im}\left(\Psi_{S}^{T}\right)\right)+r_{\varphi}\left(\mathrm{E}_{\mathbf{L}}^{T}\right)-r_{\varphi} \mu_{p}(\mathbf{L})=r_{\varphi}\left(\operatorname{ker}\left(\Psi_{S}^{T}\right)\right)
$$

d'où (i). Le second point s'en déduit immédiatement. $\diamond$

2.3. Les premières conséquences. - Reprenons les notations introduites précédemment.

Proposition 2.5. - Supposons \# $\Delta$ premier à p. Lorsque le groupe $H^{2}\left(\mathrm{G}_{\mathbf{M}}, \mathbb{Q}_{p} / \mathbb{Z}_{p}\right)$ est trivial, la représentation $\rho_{\mathbf{M}}$ est sans obstruction dès lors que le $\Delta$-module $\operatorname{Tor}\left(\mathrm{H}_{\mathbf{M}}^{a b}\right)$ est premier au $\Delta$-module Ad.

En particulier, si le morphisme $\Psi_{S}^{T}$ est injectif et si le $\Delta$-module $\operatorname{Tor}\left(\mathrm{P}_{S}^{T, a b}\right)$ est premier au $\Delta$ module $\mathrm{Ad}$, la représentation $\rho_{S}^{T}$ est sans obstruction (c'est ce que l'on peut appeler la situation "prime-to-adjoint").

Démonstration. - C'est le lemme 1.3 associé au corollaire 2.4.

$\diamond$

Comme nous l'avons vu, quand le groupe $H^{2}\left(\mathrm{H}_{\mathbf{M}}, \mathbb{Q}_{p} / \mathbb{Z}_{p}\right)$ est trivial, le groupe de cohomologie $H^{2}\left(\mathrm{G}_{\mathbf{M}}, \mathrm{Ad}\right)$ s'interprète à partir de la torsion de $\mathrm{H}_{\mathbf{M}}^{a b}$. Lorsque $\mathbf{M}=\mathbf{L}_{S}^{T}(p)$, ce groupe de torsion a déja beaucoup été étudié.

Proposition 2.6 (Gras [8], Théorème 2.6 page 261). - Si la p-partie $\mathrm{Cl}_{\mathbf{L}}^{T}(p)$ du groupe des $T$-classes de $\mathbf{L}$ est triviale, alors l'application d'Artin permet d'obtenir l'isomorphisme suivant :

$$
\operatorname{Tor}\left(\mathrm{P}_{S}^{T, a b}\right) \simeq \operatorname{Tor}\left(\mathfrak{U}_{S} / \Psi_{S}^{T}\left(\mathbb{Z}_{p} \otimes E_{\mathbf{L}}^{T}\right)\right) .
$$

Corollaire 2.7 (Gras [8] Théorème 2.6 page 261 et lemme 4.2.4 page 295)

Le pro-p-groupe $\mathrm{P}_{S}^{T, a b}$ est sans torsion dès lors que $\mathrm{Cl}_{\mathbf{L}}^{T}$ est d'ordre premier à $p$, que $\sum_{v \in S} \mu_{v} / \mu_{\mathbf{L}}=$ 0 et que $\operatorname{Tor}\left(\log \left(\mathfrak{U}_{S}\right) / \mathbb{Z}_{p} \log \left(\Psi_{S}^{T}\left(E_{\mathbf{L}}^{T}\right)\right)=0\right.$.

Pour être complet, citons la remarque suivante :

Remarque 2.8 (Wingberg, [19]). - Pour que le groupe $H^{2}\left(\mathrm{P}_{S}, \mathbb{F}_{p}\right)^{\Delta}$ soit trivial, il est nécessaire que l'extension $\mathbf{K}_{S}(p) / \mathbf{K}$ soit pro-p-libre. 


\section{Sur la dimension de Krull de l'anneau $\mathrm{R}_{S}^{T}$}

3.1. Cas où $\# \Delta$ est une puissance de $p$. -

Proposition 3.1. - Supposons que $\Delta$ est un p-groupe et que pour toute place $v$ de $T, \Delta_{v}=\{1\}$. Alors

$$
\operatorname{dimkrull}\left(\mathbf{R}_{S}^{T} / p \mathbf{R}_{S}^{T}\right) \geq 1+4\left(\delta_{S}-\left(r_{1}+r_{2}+|T|\right)\right) .
$$

Démonstration. - Si pour un groupe profini $G$, l'on dénote par $\chi_{2}(G)$ sa caractéristique d'EulerPoincaré tronquée à l'ordre 2 , il est bien connu que

$$
\chi_{2}(G, A) \leq \# A \cdot \chi_{2}\left(G, \mathbb{F}_{p}\right),
$$

où $A$ est un $G$-module fini.

Ensuite (cf. [7], [14], $\cdots$ ou propositions 2.1 et 2.2 )

$$
\chi_{2}\left(\mathrm{G}_{S}^{T}(p), \mathbb{F}_{p}\right) \leq r_{1}+r_{2}+|T|-\delta_{S},
$$

où $G_{S}^{T}(p)=\operatorname{Gal}\left(\mathbf{K}_{S}^{T}(p) / \mathbf{K}\right)$. Comme $\mathbf{L}_{S}^{T}(p)=\mathbf{K}_{S}^{T}(p)$, il vient

$$
\operatorname{dimkrull}\left(\mathrm{R}_{S}^{T} / p \mathbf{R}_{S}^{T}\right) \geq 1+4\left(\delta_{S}-\left(r_{1}+r_{2}+|T|\right)\right),
$$

$\mathrm{R}_{S}^{T}$ étant l'anneau de déformation associé à l'extension $\mathbf{L}_{S}^{T}(p) / \mathbf{K} . \diamond$

3.2. Situation modérée (preuve du théorème 0.5). — Les propositions 2.1 et 2.2 permettent de montrer l'inégalité suivante :

$$
r_{\varphi}\left(H^{1}\left(\mathrm{P}_{S}^{T}, \mathbb{F}_{p}\right)^{*}\right)-r_{\varphi}\left(H^{2}\left(\mathrm{P}_{S}^{T}, \mathbb{F}_{p}\right)^{*}\right) \geq<\mathbf{1}, \varphi>+\delta_{S} \varphi(1)-\sum_{v \in T \cup P l_{\infty}} r_{\varphi}\left(\operatorname{Ind}_{\Delta_{v}}^{\Delta} \mathbf{1}\right) .
$$

Notons ensuite l'identité suivante :

$$
\begin{aligned}
& \delta_{S} \varphi(1)-\sum_{v \in T \cup \infty}<\operatorname{Ind}_{\Delta_{v}}^{\Delta} 1, \varphi> \\
& =\left(\delta_{S}-\left(r_{1}+r_{2}+|T|\right)\right) \varphi(1)+\sum_{v \in T \cup P l_{\infty}}<\varphi,\left(\operatorname{Ind}_{\Delta_{v}}^{\Delta} \operatorname{Ind}_{\{\mathbf{1}\}}^{\Delta_{v}} \mathbf{1}-\operatorname{Ind}_{\Delta_{v}}^{\Delta} \mathbf{1}\right)>.
\end{aligned}
$$

Comme $\operatorname{Ind}_{\{\mathbf{1}\}}^{\Delta_{v}} \mathbf{1}$ contient la représentation triviale, la quantité

$$
\sum_{v \in T \cup P l_{\infty}}<\varphi,\left(\operatorname{Ind}_{\Delta_{v}}^{\Delta} \operatorname{Ind}_{\{1\}}^{\Delta_{v}} \mathbf{1}-\operatorname{Ind}_{\Delta_{v}}^{\Delta} 1\right)>
$$

est positive.

Ainsi sous la condition $\delta_{S} \geq\left(r_{1}+r_{2}+|T|\right)$, il vient pour tout caractère $\mathbb{C}_{p}$-irréductible $\varphi$ l'inégalité suivante :

$$
r_{\varphi}\left(H^{1}\left(\mathrm{P}_{S}^{T}, \mathbb{F}_{p}\right)^{*}\right)-r_{\varphi}\left(H^{2}\left(\mathrm{P}_{S}^{T}, \mathbb{F}_{p}\right)^{*}\right) \geq 0 \text {. }
$$

Regardons maintenant les termes locaux.

Pour $v \mid \infty$ décomposée dans $\mathbf{L} / \mathbf{K}$, il vient

$$
\operatorname{Ind}_{\Delta_{v}}^{\Delta} \operatorname{Ind}_{\{1\}}^{\Delta_{v}} 1-\operatorname{Ind}_{\Delta_{v}}^{\Delta} 1=0 .
$$

Pour $v \mid \infty$ paire, non-décomposée dans $\mathbf{L} / \mathbf{K}$, il vient

$$
\operatorname{Ind}_{\{1\}}^{\Delta_{v}} \mathbf{1}=\mathbf{1}+\chi_{v}
$$

où $\chi_{v}$ est l'unique caractère irréductible non trivial de $\Delta_{v}$. Et ainsi, pour cette même place,

$$
<\operatorname{Ind}_{\Delta_{v}}^{\Delta} \chi_{v}, \operatorname{Ad}>=<\chi_{v}, \operatorname{Ad}>_{\mid \Delta_{v}}=<\chi_{v}, 4 \cdot 1>_{\mid \Delta_{v}}=0 .
$$

Pour $v \mid \infty$ impaire :

$$
<\operatorname{Ind}_{\Delta_{v}}^{\Delta} \chi_{v}, \operatorname{Ad}>=<\chi_{v}, \operatorname{Ad}>_{\mid \Delta_{v}}=<\chi_{v}, 2 \cdot \mathbf{1}+2 \cdot \chi_{v}>_{\mid \Delta_{v}}=2 .
$$


Notons par $\sum_{\varphi} \alpha_{\varphi} \varphi$ la décomposition de la représentation adjointe Ad en somme de caractères $\mathbb{C}_{p}[\Delta]$-irréductibles. Pour $v$ impaire, il vient finalement

$$
\sum_{\varphi} \alpha_{\varphi}<\varphi, \chi_{v}>_{\mid \Delta_{v}}=2 .
$$

En regroupant tout ceci, on obtient alors

$$
\begin{aligned}
< & \sum_{\varphi}\left(r_{\varphi}\left(H^{1}\left(\mathrm{P}_{S}^{T}, \mathbb{F}_{p}\right)^{*}\right)-r_{\varphi}\left(H^{2}\left(\mathrm{P}_{S}^{T}, \mathbb{F}_{p}\right)^{*}\right)\right) \varphi, \operatorname{Ad}> \\
= & <\sum_{\varphi}\left(r_{\varphi}\left(H^{1}\left(\mathrm{P}_{S}^{T}, \mathbb{F}_{p}\right)^{*}\right)-r_{\varphi}\left(H^{2}\left(\mathrm{P}_{S}^{T}, \mathbb{F}_{p}\right)^{*}\right)\right) \varphi, \sum_{\varphi} \alpha_{\varphi} \varphi> \\
\geq & \left(\sum_{\varphi} \alpha_{\varphi}\left(<\mathbf{1}, \varphi>+\left(\delta_{S}-r_{1}+r_{2}+|T|\right) \varphi(1)\right)\right)+ \\
& \sum_{T \cup P l_{\infty}} \sum_{\varphi} a_{\varphi}<\varphi, \operatorname{Ind}_{\Delta_{v}}^{\Delta} \operatorname{Ind}_{\mathbf{1}}^{\Delta_{v}} \mathbf{1}-\operatorname{Ind}_{\Delta_{v}}^{\Delta} \mathbf{1}> \\
= & \left(\sum_{\varphi} \alpha_{\varphi}\left(<\mathbf{1}, \varphi>+\left(\delta_{S}-r_{1}+r_{2}+|T|\right) \varphi(1)\right)\right)+ \\
& \sum_{v \mid \infty, \Delta_{v} \neq 1} \sum_{\varphi} \alpha_{\varphi}<\chi_{v}, \varphi>_{\mid \Delta_{v}}+\sum_{v \in T}\left(<\operatorname{Ad}, \operatorname{Ind}_{\{1\}}^{\Delta} \mathbf{1}>-\left\langle\operatorname{Ad}, \operatorname{Ind}_{\Delta_{v}}^{\Delta} \mathbf{1}\right\rangle\right) \\
= & a_{1}+\left(\delta_{S}-\left(r_{1}+r_{2}+|T|\right)\right) \sum_{\varphi} \alpha_{\varphi} \varphi(1)+2 \kappa_{1}+\sum_{v \in T} \kappa_{v} \\
= & a_{1}+4\left(\delta_{S}-\left(r_{1}+r_{2}+|T|\right)\right)+2 \kappa_{1}+\kappa_{T} .
\end{aligned}
$$

Pour conclure, on a le lemme suivant :

Lemme 3.2. - Soient $M$ et $N$ deux $\mathbb{Z}_{p}[\Delta]$-modules. Alors le p-rang de $(M \otimes N)^{\Delta}$ est égal au produit scalaire $\left\langle M^{*}, N\right\rangle$.

En effet, le $p$-rang de $(M \otimes N)^{\Delta}$ est égal exactement au nombre de fois qu'apparait le représentation triviale dans $M \otimes N$. Ainsi, si $M=\sum_{\psi} \beta_{\psi} \psi$ et $N=\sum_{\varphi} \beta_{\varphi}^{\prime} \varphi$ sont les décompositions de $M$ et $N$ en caractères $\mathbb{C}_{p}$-irréductibles, il vient :

$$
\begin{aligned}
<\mathbf{1}, M \otimes N> & =\sum_{\varphi, \psi} \beta_{\psi} \beta_{\varphi}^{\prime}<\mathbf{1}, \varphi \psi> \\
& =\sum_{\varphi, \psi} \beta_{\psi} \beta_{\varphi}^{\prime}<\varphi^{-1}, \psi> \\
& =<M^{*}, N>
\end{aligned}
$$

On obtient de tout ceci une minoration de la dimension de Krull de $\mathrm{R}_{S}^{T} / p \mathrm{R}_{S}^{T}$ dès lors que l'on aura noté que celle-ci vaut au moins

$$
\sum_{\varphi}<\left(r_{\varphi}\left(H^{1}\left(\mathrm{P}_{S}^{T}, \mathbb{F}_{p}\right)^{*}\right)-r_{\varphi}\left(H^{2}\left(\mathrm{P}_{S}^{T}, \mathbb{F}_{p}\right)^{*}\right)\right) \varphi, \mathrm{Ad}>
$$

(car chaque terme est positif).

En remarque, notons que $a_{1} \geq 1$ et que si $\operatorname{Im}(\bar{\rho})$ est un sous-groupe de Cartan, la représentation adjointe Ad contient au moins deux fois la représentation unité $\mathbf{1}$ (et donc que $a_{\mathbf{1}}>1$ ). $\diamond$

Corollaire 3.3. - Supposons que $S$ contient l'ensemble des places au-dessus de $p$ et prenons $T=\emptyset$. Alors

$$
\operatorname{dimkrull}\left(\mathrm{R}_{S}\right) \geq 1+2 \kappa_{1}+4 r_{2} .
$$

\section{Détail d'un exemple}

Comme indiqué dans l'introduction, l'exemple suivant s'inspire de l'exemple décortiqué par Mazur dans $[\mathbf{1 5}]$.

Fixons un plongement $\overline{\mathbb{Q}} \hookrightarrow \mathbb{C}$.

Soit un entier $a>0$. Soit le polynôme irréductible $P(x)=x^{3}+a x+1$ de discriminant $d=-4 a 3-27$ (que l'on souhaite sans facteur carré) et ayant pour groupe de Galois $S_{3}$.

Considérons les corps suivants : $\mathbf{K}=\mathbb{Q}(\sqrt{d}) ; \mathbf{F}=\mathbb{Q}(\theta)$, où $\theta$ est la racine réelle de $P ; \mathbf{L}=\mathbb{Q}(\sqrt{d}, \theta)$. Soit $\theta^{\prime}$ une seconde racine de $P$. 
L'extension $\mathbf{L} / \mathbf{K}$ est non-ramifiée, en particulier $\mathbf{L}$ est contenue dans $\mathbf{K}_{S} / \mathbf{K}$, et ce pour tout ensemble $S$.

Soit $\bar{\rho}: \Delta \hookrightarrow \mathrm{Gl}_{2}\left(\mathbb{F}_{\mathrm{p}}\right)$ une représentation injective de $\Delta$ dans $\mathrm{Gl}_{2}\left(\mathbb{F}_{\mathrm{p}}\right)$, pour un certain nombre premier $p$. L'image $\bar{\rho}(\Delta)$ est un sous-groupe de Cartan (qui est déployé si et seulement si, 3 divise $p-1$ ). Nous souhaitons caractériser des situations où l'anneau de déformation de $\bar{\rho}$ est sans obstruction.

Commençons par regarder les unités du corps $\mathbf{L}$.

Lemme 4.1. - (i) L'élément $\theta$ (resp. $\left.\theta^{\prime}\right)$ est une unité fondamentale de $\mathbf{F}$ (resp. de $\mathbb{Q}\left(\theta^{\prime}\right)$ ).

(ii) Pour $p>3$, la famille $\left\{\theta, \theta^{\prime}\right\}$ forme une $\mathbb{Z}_{p}$-base de $\mathrm{E}_{\mathbf{L}}$.

Démonstration. - (i) Soit $a>1$ (le cas $a=1$ se traite directement).

Soit $\operatorname{Reg}(\mathbf{F})$ le régulateur du groupe des unités $E_{\mathbf{F}}$ de $\mathbf{F}$. Notons par $\operatorname{Reg}(\theta)=|\log | \theta||$ le régulateur du sous réseau $\theta^{\mathbb{Z}}$ de $E_{\mathbf{F}}$.

D'après Cusick [5], on connait une minoration $\operatorname{de} \operatorname{Reg}(\mathbf{F})$ :

$$
\operatorname{Reg}(\mathbf{F}) \geq \frac{1}{3} \log ((4 a 3+27) / 27)
$$

car $4 a 3+27$ est sans facteur carré.

Ainsi

$$
\left[E_{\mathbf{F}}:< \pm \theta>\right]=\operatorname{Reg}(\theta) / \operatorname{Reg}(\mathbf{F})=\frac{3|\log | \theta||}{\log ((4 a 3+27) / 27)} .
$$

On note ensuite que $\theta \in[-1 / a ;-1 / a 2]$ et est d'autant plus proche de $-1 / a$ que $a$ est grand. Il vient alors facilement que $\operatorname{Reg}(\theta) / \operatorname{Reg}(\mathbf{F})<2$ et donc que $\mathrm{E}_{\mathbf{F}}=< \pm \theta>$.

(ii) Remarquons tout d'abord, d'après (i), que si $\theta$ est divisible alors nécessairement $\mathbf{L}=\mathbf{F}(\sqrt{ \pm \theta})$. Comme $\mathbf{L}=\mathbf{F}(\sqrt{-4 a 3-27})$, par la théorie de Kummer,

$$
\pm \theta(-4 a 3-27)^{-1} \in \mathbf{F} 2,
$$

ce qui est impossible (la contradiction est apportée par un premier $l$ divisant $4 a 3+27$ ).

Ainsi $\theta$ n'est pas divisble. Soit alors $\{\theta, \xi\}$ une $\mathbb{Z}$-base de $\mathrm{E}_{\mathbf{L}}$. Ainsi $N_{\mathbf{L} / \mathbf{F}} \xi= \pm \theta^{\varepsilon+2 n}$ avec $\varepsilon=0$ ou 1 et $n \in \mathbb{Z}$. Par conséquent, $N_{\mathbf{L} / \mathbf{F}}\left(\xi \theta^{-n}\right)= \pm \theta^{\varepsilon}$. La famille $\left\{\theta, \xi \theta^{-n}\right\}$ formant également une base de $\mathrm{E}_{\mathbf{L}}$, on peut donc supposer que $N_{\mathbf{L} / \mathbf{F}} \xi= \pm \theta^{\varepsilon}$.

Écrivons ensuite $\theta^{\prime}$ dans la base $\{\theta, \xi\}$. Il existe $\alpha, \beta \in \mathbb{Z}$, tels que $\theta^{\prime}= \pm \theta^{\alpha} \xi^{\beta}$. Prenons la norme dans $\mathbf{L} / \mathbf{F}$ de cette égalité :

$$
-\theta^{-1}=N_{\mathbf{L} / \mathbf{F}} \theta^{\prime}= \pm \theta^{2 \alpha+\beta \varepsilon}
$$

ainsi $\varepsilon=1$ et il vient l'équation $-1=2 \alpha+\beta$. Regardons la norme de $\theta^{\prime}$ dans $\mathbf{L} / \mathbb{Q}\left(\theta^{\prime}\right)$ :

$$
\theta^{\prime} 2= \pm \theta^{\prime-\alpha+\lambda \beta}
$$

où $N_{\mathbf{L} / \mathbb{Q}\left(\theta^{\prime}\right)} \xi= \pm \theta^{\prime \lambda}$. D'où l'équation $2=-\alpha+\lambda \beta$. De ces deux dernières équations, on en déduit

$$
3=\beta(1+2 \lambda)
$$

ainsi $\beta \mid 3$ et le second point du lemme s'en déduit. $\diamond$

Choisissons un nombre premier $p>3$ totalement décomposé dans $\mathbf{F} / \mathbb{Q}$ (ou de façon équivalente totalement décomposé dans $\mathbf{L} / \mathbf{K})$. Assurons-nous que $p$ est premier à l'ordre du groupe des classes de $\mathbf{L}$ et que $p$ ne divise pas $d$.

Soit $\left(x-u_{1}\right)\left(x-u_{2}\right)\left(x-u_{3}\right)$, avec $u_{i} \neq u_{j}$, la factorisation de $P$ dans $\mathbb{F}_{p}[x]$.

Prenons $T=\emptyset$ et $S=\{v\}$, où $v$ est l'une des deux places de $\mathbf{K}$ au-dessus de $p$. 
Dans ce contexte, le morphisme $\Psi_{S}: \mathbb{Z}_{p} \otimes \mathrm{E}_{L} \rightarrow \mathfrak{U}_{S(\mathbf{L})}$ est injectif (cf. par exemple [10], [14]). Ainsi le groupe $H^{2}\left(\mathrm{P}_{S}, \mathbb{Q}_{p} / \mathbb{Z}_{p}\right)$ est trivial (cf. corollaire 2.4).

Nous allons maintenant imposer des conditions afin que la torsion $\operatorname{Tor}\left(\mathrm{P}_{S}^{a b}\right)$ de $\mathrm{P}_{S}^{a b}$ soit également triviale.

Soient $w_{(i)}$ les trois places de $\mathbf{L}$ au-dessus de $v$.

Il est facile de noter que chaque premier de $\mathbf{F}$ au-dessus de $p$ donne lieu à une place de $S(\mathbf{L})$.

Assurons-nous que $\pi_{i}=\theta-u_{i}$ est une uniformisante de $\mathbf{L}_{w_{(i)}}$ (avec $\left.u_{i} \in\right] 0, p-1[$ ). Ceci se lit dans le corps $\mathbf{F}$.

Posons $e_{i}=\log _{w_{i}}(\theta)$. Alors $\log \mathfrak{U}_{S}=\mathbb{Z}_{p} e_{1} \oplus \mathbb{Z}_{p} e_{2} \oplus \mathbb{Z}_{p} e_{3}$ et $\log \left(\theta^{\prime}\right)=\sum_{i} \lambda_{i} e_{i}$, avec $\lambda_{i} \in \mathbb{Z}_{p}$. Il est facile de voir que l'on peut s'arranger pour que $\theta^{\prime}$ vérifie :

$$
\left\{\begin{array}{l}
\theta^{\prime}=u_{3}+\pi_{1} \\
\theta^{\prime}=u_{1}+\pi_{2} \\
\theta^{\prime}=u_{2}+\pi_{3}
\end{array}\right.
$$

Soit donc $y=\sum_{i} y_{i} e_{i}$ un élément de $p$-torsion de $\log \left(\mathfrak{U}_{S}\right) / \log \left(\mathrm{E}_{\mathbf{L}}\right)$. Il existe $a, b \in \mathbb{Z}_{p}$ tels que

$$
p\left(y_{1} e_{1}+y_{2} e_{2}+y_{3} e_{3}\right)=a\left(e_{1}+e_{2}+e_{3}\right)+b\left(\lambda_{1} e_{1}+\lambda_{2} e_{2}+\lambda_{3} e_{3}\right),
$$

d'où le système linéaire

$$
\left\{\begin{array}{l}
p y_{1}=a+b \lambda_{1} \\
p y_{2}=a+b \lambda_{2} \\
p y_{3}=a+b \lambda_{3}
\end{array}\right.
$$

Ainsi, et c'est notre dernière condition sur $p$, s'il existe $i, j$ tels que $\lambda_{i} \neq \lambda_{j}(\bmod p)$, il vient immédiatement $b \equiv a \equiv 0(\bmod p)$, c'est à dire $y \in \Psi_{S}\left(\mathrm{E}_{\mathbf{L}}\right)$ et de ce fait la $p$-torsion de $\mathfrak{U}_{S} / \Psi_{S}\left(\mathrm{E}_{L}\right)$ est triviale.

Interprétons un peu plus précisémment cette condition. On a

$$
\begin{aligned}
\lambda_{1} & =\log _{w_{1}}\left(\theta^{\prime}\right) / \log _{w_{1}}(\theta) \\
& =\frac{u_{1}}{u_{3}} \bmod \pi_{1} \\
\lambda_{2} & =\frac{u_{2}}{u_{1}} \bmod \pi_{2} \\
\lambda_{3} & =\frac{u_{3}}{u_{2}} \bmod \pi_{3}
\end{aligned}
$$

De ce fait la $p$-torsion de $\mathfrak{U}_{S} / \Psi_{S}\left(\mathrm{E}_{L}\right)$ est triviale dès lors que pour un triplet $(i, j, k), i, j, k$ deux à deux différents, on a

$$
u_{i} u_{j} \neq u_{k} 2
$$

Si tel est le cas, le corollaire 2.7 s'applique. Le pro-p-groupe $\mathrm{P}_{S}^{a b}$ est sans torsion.

Comme $H^{2}\left(\mathrm{P}_{S}, \mathbb{Q}_{p} / \mathbb{Z}_{p}\right)=0$, on en déduit que $\mathrm{P}_{S}$ est libre et par conséquent $H^{2}\left(\mathrm{P}_{S}, \mathrm{Ad}\right)=0$. L'anneau $\mathrm{R}_{S}$ est sans obstruction.

Le nombre minimal de générateurs de $\mathrm{R}_{S}$ est exactement égal à la dimension sur $\mathbb{F}_{p}$ de $H^{1}\left(G_{S}, \mathrm{Ad}\right)$, et $H^{1}\left(G_{S}, \mathrm{Ad}\right) \simeq\left[\mathrm{P}_{S}^{a b^{*}} \otimes \mathrm{Ad}\right]^{\Delta}$. Or $\Delta$ agit trivialement sur $\mathrm{P}_{S}^{a b} \simeq \mathbb{Z}_{p}$ et Ad contient deux fois le caractère trivial 1. Par conséquent $\mathrm{R}_{S}=\mathbb{Z}_{p}\left[\left[T_{1}, T_{2}\right]\right]$. 
Le groupe $\mathrm{P}_{S}$, isomorphe à $\mathbb{Z}_{p}$, est engendré par un élément $x$. Alors $\operatorname{Gal}\left(\mathbf{L}_{S}(p) / \mathbf{K}\right) \simeq \Delta \times\langle x\rangle$ et $\rho_{S}$ peut être définie par :

$$
\rho_{S}(x)=\left(\begin{array}{cc}
1+T_{1} & 0 \\
0 & 1+T_{2}
\end{array}\right) .
$$

Ceci, par exemple, s'applique aux couples

$$
(a, p)=(2,17),(2,71), \cdots,(4,71),(4,73),(4,83), \cdots,(5,19),(5,29),(5,73), \cdots
$$

\section{Références}

[1] G. Böckle, Explicit universal deformations of even Galois represnetations, Math. Nach. 206 (1999), 85-110.

[2] G. Böckle, A. Mézard, The prime-to-adjoint principle and unobstructed Galois deformations in the Borel case, Journal of Number Theory 78 (1999), 167-203.

[3] N. Boston, Explicit deformation of Galois representations, Invent. math. 103 (1990), 181-186.

[4] N. Boston, B. Mazur, Explicit universal deformations of Galois representations, Adv. Stud. Pure Math. 17 (1989), 1-21.

[5] T.W. Cusick, Lower bounds for regulators, Number Theory Noordwijkerhout, Lecture Notes in Math. 1068, 1983.

[6] F. Gouvêa, Deforming Galois Representation : a survey, Canadian Math. Society, Conference proceeding 17 (1995).

[7] G. Gras, Théorèmes de réflexion, Journal Th. des Nombres de Bordeaux 10 (1988), 399-499.

[8] G. Gras, Class Field Theory, From Theory to practice, Springer-Verlag, Berlin, 2003.

[9] K. Haberland, Galois cohomology of algebraic number fields. VEB Deutscher Verlag der Wissenschaften, Berlin, 1978.

[10] J.-F. Jaulent, Sur l'indépendance $l$-adique de nombres algébriques, J. Number Theory 20 (1985), 149-158.

[11] H. Kisilevsky, J. Labute, On a sufficient condition for the p-class tower of a CM-field to be infinite, Théorie des nombres (Quebec, PQ, 1987), 556-560, de Gruyter, Berlin, 1989.

[12] H. Koch, Galoissche Theorie des p-Erweiterungen, VEB, Berlin, 1970.

[13] C. Maire, Compléments à un résultat de Shafarevich, Math. Nachr. 198 (1999), 149-168.

[14] C. Maire, On $\mathbb{Z}_{l}$-rank of abelian extensions with restricted ramification, Journal of Number Theory 92 (2002), 376-404.

[15] B. Mazur, Deforming Galois representations In : Galois groups over $\mathbb{Q}$, Y. Ihara, K. Ribet, J.-P. Serre eds., MSRI Publ. 16, Springer-Verlag, 1987, 385-437.

[16] A. Mézard, Computation of a universal deformation ring in the Borel case, Math. Proc. Cambridge Philo. Soc. 126 (1999), 417-442.

[17] J. Neukirch, A. Schmidt, K. Wingberg, Cohomology of Number Fields, GMW 323, Springer-Verlag Berlin Heidelberg, 2000.

[18] R. Ramakrishna, Deforming an even representation, Invent. math. 132 (1998), 563-580.

[19] K. Wingberg, Free pro-p-extensions of number fields, preprint 2005.

\section{7 novembre 2006}

Christian Maire, Institut de Mathématiques de Toulouse, Université Toulouse Le Mirail, 5 allées Machado, 31058 Toulouse $\bullet$ E-mail : maire@univ-tlse2.fr 